\title{
Structure and Function of the Perinucleolar Compartment in Cancer Cells
}

\author{
A. SlusarczyK, ${ }^{1}$ R. Kamath,${ }^{1}$ C. Wang,,${ }^{1}$ D. Anchel,${ }^{2}$ C. Pollock,,${ }^{1}$ M.A. Lewandowska,,${ }^{1}$ \\ T. FitZPATRICK, ${ }^{1}$ D.P. BAZETT-JONES, ${ }^{2}$ AND S. HUANG ${ }^{1}$ \\ ${ }^{1}$ Department of Cell and Molecular Biology, Northwestern University Feinberg School of Medicine, Chicago, Illinois \\ 60614; ${ }^{2}$ Department of Biochemistry, University of Toronto, Toronto, Canada M5G 1L7 \\ Correspondence: s-huang2@northwestern.edu
}

\begin{abstract}
The perinucleolar compartment (PNC) is a subnuclear body that forms in cancer cells. In vivo analyses using human tumor tissues demonstrate a close correlation between PNC prevalence and disease progress in colorectal carcinoma, and a high PNC prevalence is associated with poor patient outcome. These findings are consistent with previous observations in breast cancer and cancer cell lines in vitro. The PNC is composed of thick strands that form a filamental meshwork often extending into the nucleolus. Although it appears to be electron dense as observed by transmission electron microscopy (TEM), the actual density of the structure imaged by electron spectroscopy is much lower, similar to that of the interchromatin space, and is lined with ribonucleoproteins (RNPs). In situ detections show that the PNC is highly enriched with a subset of small RNAs of polymerase III (Pol III) origins and RNA-binding proteins primarily implicated in pre-mRNA processing. A novel gelshifting approach demonstrates that the addition of PNC-associated RNAs into HeLa cell lysates increases the mobility of polypyrimidine tract-binding (PTB) protein in a native gel electrophoresis, suggesting an interaction between these RNAs and PTB proteins. On the basis of these and other findings, we propose a working model in which novel RNPs have a key role in regulating gene expression at the PNC in cancer cells.
\end{abstract}

The nucleus is a highly organized and compact structure where essential cellular functions take place, including DNA replication, transcription, RNA processing, metabolism, noncoding RNA function, macromolecular transport, and regulation of all of these processes. During the past few decades, the structure of nuclear bodies and their functional relevance to gene expression have been increasingly explored and described. Classic nuclear bodies have been investigated, but many new nuclear domains have also been described. One type of nuclear body, including the nucleolus, Cajal body, chromocenter, bar body, and perinucleolar compartment, represents those that have distinguishing morphological structures detectable by either light or electron microscopy without specific probe labeling. Another increasingly described category of nuclear bodies is that of nuclear domains, detectable only through specific labeling of proteins or RNAs. Study of molecular features of nuclear bodies reveals that they are often enriched with factors involved in one or multiple functions. One of the most studied nuclear bodies is the nucleolus, in which ribosome synthesis and export have been identified to take place. More recently, other functions including chromatin remodeling, transport, regulation of other nuclear factors through sequestration, and aging have also been shown to associate with the nucleolus (Pederson 1998). Extensive investigation has also been devoted to the understanding of nuclear bodies including promyelocytic leukemia (PML) nuclear bodies, the Cajal, and chromocenters (Lamond and Earnshaw 1998; Gall 2003; Lamond and Spector 2003; Spector 2003). Although each body seems to be involved in more than one function, their precise cellular activities and functional significance remain to be clarified.

Although many of nuclear bodies are conserved, several of them appear to be nonessential to cell viability because the perturbation of the structures is often not lethal to cells, as long as the functions that associate with these bodies remain sufficiently active. For example, disruption of nucleoli by deletion of nucleolus organizing regions (NORs) in yeast does not affect cell viability when ribosomal DNA (rDNA) is transcribed from exogenous plasmids by a Pol II promoter (Oakes et al. 1999). Loss of Cajal bodies in mouse or Drosophila has little consequence in surviving animals (Tucker et al. 2001; Liu et al. 2009). Although a deletion of coilin in zebrafish is lethal (Strzelecka et al. 2010), it is not clear whether it is the coilin or the Cajal body that is important for zebrafish. Therefore, why do cells assemble these nonmembranebound nuclear bodies? What might be the functional significance for factors involved in various functions to be concentrated at a specific nuclear space? It can be speculated that the nucleation of factors involved in specific functions could enhance the efficiency of corresponding processes. Experiments are needed to test this hypothesis.

The PNC is a structure that physically associates with the nucleolus but contains little to no known nucleolar factors or newly synthesized ribosomes. The formation of the structure is the result of advanced transformation, and we believe that it may represent a physiological adaptation of cells to help maintain and sustain the malignant state of cancer cells. Here, we describe our current understanding regarding the structure and function of the PNC. 


\section{STRUCTURE OF THE PNC}

The PNC can be visualized at the light microscopic level by immunolabeling with SH54, an antibody that specifically recognizes the PTB protein, a bona fide component of the PNC (Fig. 1A). The PNC can also be detected by TEM through conventional uranyl acetate (UA)-lead staining (Fig. 1B). When 50-nm thin sections of cells were examined by TEM, the PNC was observed to be a dense structure organized in a reticulated filamental meshwork either at the surface of (Fig. 1B, outlined area) or often extended into the nucleolus. Although it appears similar in density to nucleoli in regular EM staining (Fig. 1B) as examined by TEM, the actual density is very different when examined by electron spectroscopic imaging (ESI). ESI allows for the mapping of intracellular distribution of elements nitrogen or phosphates at electromicroscopic resolution. Because these elements are differentially represented in different macromolecules, such as nucleic acid or proteins, the imaging allows for the identification of various nuclear compositions. HeLa cells were prepared for ESI based on the established protocol described by Dellaire et al. 2004. The area occupied by the PNC is identified by immunogold labeling with anti-PTB antibody (Fig. 1C, round blue beads). As shown in Figure 1C, the PNC extends into the nucleolus as a filamental network and is low in density, similar to that of interchromatin space. The PNC contains strands of blue (nitrogen) touched with yellow (phosphate), a signature of RNPs (Fig. 1C, arrowheads). There is little to no dense heterochromatin (dense yellow areas) at the nucleolar surface where the PNC attaches to the nucleolus. In combination with the UA-lead-stained EM micrograph (Fig. 1B), we conclude that the PNC is organized in an extended meshwork with fibril structures physically associated with the nucleolus. The PNC is similar in density to the nucleoplasm and is highly enriched with RNA-protein complexes.

One explanation for the physical association between the PNC and the nucleolus is that the PNC associates with nucleolar-linked DNA. Evidence of the association of the PNC with DNA is provided by our recently published studies (Norton et al. 2009), in which we demonstrated that the base-pairing capacity of the DNA structure is critical for the maintenance of the PNC, whereas strand breaks and alkalization have very little impact. The number of PNCs increases correspondingly with the number of endoreplication cycles of CDK1 conditional mutant cells at a nonpermissive temperature. In addition, the PNC splits during DNA replication in a manner similar to that of a DNA locus. These findings argue strongly that the PNC is nucleating on a DNA locus or loci. It is possible that PNC-associated DNA is part of or near to NORs that contain rDNA clusters and are the core for the nucleolar assembly. Alternatively, the locus could be part of the perinucleolar heterochromatin that is transcribed by Pol III and that is normally less or not active in non-PNC-containing cells. Studies are underway to identify the DNA locus/loci that nucleate PNCs.

\section{THE PNC ASSOCIATES WITH MALIGNANT PHENOTYPE}

The PNC is not present in all mammalian cells. Evaluation of PNC prevalence (the percentage of nonmitotic and nonapoptotic cells containing at least one PNC) in a large number of cell lines or primary cells shows that PNCs are not detected in primary cells including human and mouse embryonic stem cells (ESCs). PNCs can be detected in a small percentage of immortalized cells, whereas PNC prevalence shows a substantial heterogeneity from $<5 \%$ to $\sim 100 \%$ in cancerous cells from patients or in cells transformed in vitro. All of these cells are derived from solid tissue origins. The vast heterogeneity leads to a hypothesis that PNC prevalence may, in some way, represent the level of malignancy in the given population of cells.

To test this hypothesis in vitro and in vivo, we have systematically examined the association of PNC prevalence
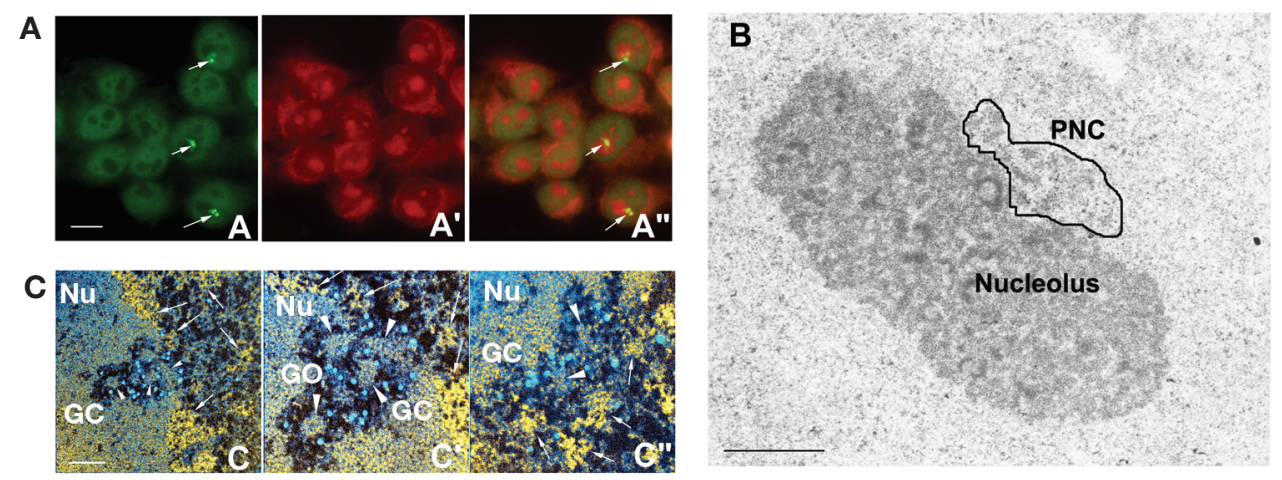

Figure 1. (A) The PNC is a perinucleolar compartment, structurally distinct from the nucleolus. PNCs $(A$, arrows, immunolabeled with the anti-PTB antibody) associate with the nucleolus ( $A^{\prime}$, immunolabeled with antiribosome antibodies). ( $\left.A^{\prime \prime}\right)$ Merge of $A$ and $A^{\prime}$ showing the physical association between the two compartments. Bar, $10 \mu \mathrm{m}$. $(B)$ TEM analyses show PNCs to be an electron-dense filamentous structure on the surface of the nucleolus (black outline). Bar, $1 \mu \mathrm{m}$. (C) ESI analyses show that the PNCs (immunogold labeling, bright blue dots) extend into the nucleolus $(\mathrm{Nu})$ around the granular component (GC) in a thick filamental form that is much less dense than the nucleolus (dark space into the dense nucleolar region; 50-80-nm thick). The PNCs are lined with RNPs (arrowheads) and are parting the compact heterochromatin on the nucleolar surface (arrows) because the yellow patches are absent at the area where the PNC contacts the nucleolus. (C) Bar, $0.2 \mu \mathrm{m},\left(C^{\prime}\right.$ and $\left.C^{\prime \prime}\right)$ bar, $0.4 \mu \mathrm{m}$. 
and metastasis. We examined PNCs in a series of well-defined human prostate cancer cell variants with characterized levels of metastatic capacities in mouse metastatic models. The results show a tight correlation between PNC prevalence and metastatic capabilities (Norton et al. 2008). PNC prevalence reaches $\sim 100 \%$ with large and typical PNCs in highly metastatic cells that were enriched for metastasis through five rounds of selection in mice. In comparison, $<5 \%$ of localized prostate tumor cells have typical and large PNCs. These localized variants were isolated from mouse prostates after four rounds of selection from a metastatic cell line for those only grown locally. Most of the detectable PNCs in these cells are highly atypical and extremely small. These findings support the fact that PNC prevalence and the size and shape of the PNCs reflect the level of metastasis in a given population of cancer cells (Norton et al. 2008).

To test the association in vivo, we evaluated PNC prevalence in primary tumors and metastasis of several tissue origins. Investigation of more than 200 samples from patients with breast cancer reveals that PNC prevalence increases along with the progression of the disease, as specified in clinical staging (Kamath et al. 2005). PNC prevalence increases in a stepwise manner in primary tumors, lymph nodes, and distant metastasis and reaches $\sim 100 \%$ in distant metastases. These findings suggest that PNC prevalence is indicative of the severity of the disease, and PNC-containing cells have a metastatic advantage over those lacking PNCs. If the PNC is indicative of the malignant capacity of the primary tumors, we would expect that PNC prevalence in primary tumors should correlate with patient outcome. Retrospective studies using case-matched samples from patients with breast cancer (invasive ductal carcinoma) show that PNC prevalence in primary tumors positively correlates with the relapse of the disease. A high PNC prevalence in node-negative stage-I breast cancer provides prognostic information in addition to grading for the recurrence of the disease (Ka- math et al. 2005). These findings support the idea that PNC-containing cells have growth and metastatic advantages over cells without PNCs, and PNC prevalence in a given population is indicative of its metastatic capacity.

In addition to breast cancer, we have also examined colorectal carcinoma. We observed a correlative trend with increased malignancy, similar to that of breast cancer. In colorectal cancer, we examined 176 normal and cancerous colorectal tissue samples. PNC prevalence increases along with disease progression in terms of clinical stages, and a high PNC prevalence in primary tumors associates with poor patient outcome (Fig. 2). A similar observation was also made for ovarian cancers. All together, our findings suggest that PNCs are likely to be the result of transformation. The formation of the PNC may mark milestone changes that are associated with metastatic capability. Thus, the PNC is a selective tumor marker that specifically reflects the metastatic behavior of cancer cells.

\section{PNC ENRICHED WITH NOVEL RNA-PROTEIN COMPLEXES}

Studies from other investigators and from our laboratory have shown that the PNC is enriched with a subset of Pol III RNA and RNA-binding proteins that have primarily been implicated in Pol II RNA metabolism (Matera et al. 1995; Lee et al. 1996; Wang et al. 2003). Pol III RNAs found in the PNC include ribozyme RNA components RNase MRP RNA and RNase P RNA; hY1, 3, and 4 RNA; signal recognition particle (SRP) RNA; and Alu RNA. Not all Pol III RNAs are found in the PNC; neither 5S RNA nor U6 RNA is detected there. Extensive search for pre-mRNA has not yielded any specific enrichment of these RNAs in the PNC, nor have ribosomal RNAs (rRNAs) transcribed by Pol I been identified at the PNC. Therefore, PNC-associated RNAs are primarily a subset of Pol III noncoding RNA. Proteins detected to be enriched in the PNC include PTB, CUGBP, KSRP, Raver1,
A

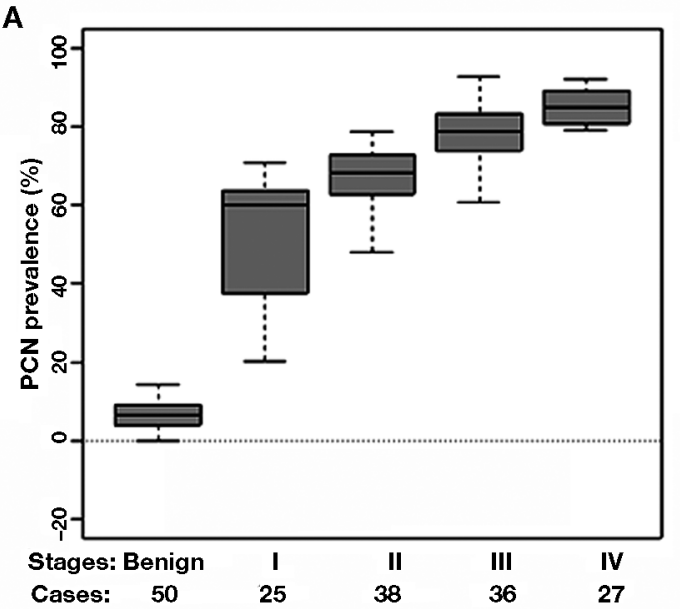

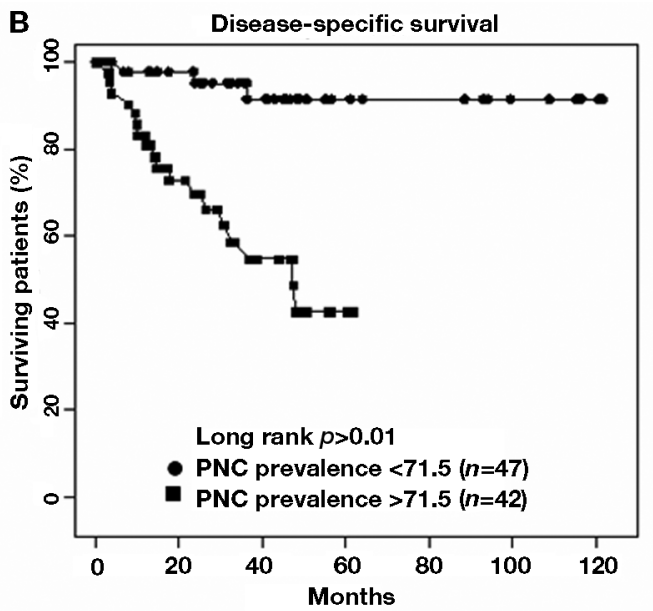

Figure 2. PNC prevalence is positively correlated with disease progression of colorectal cancers. $(A)$ PNC prevalence increases significantly in primary tumors corresponding to the increases in the stages of disease. (B) Kaplan-Meier overall survival analysis for all carcinoma patients using PNC prevalence data. Median patient follow-up time was 120 mo. These data show that a high PNC prevalence in a primary tumor associates with poor patient prognosis. 
Raver2, Rod1, and nucleolin (Timchenko et al. 1996; Huang et al. 1998; Huttelmaier et al. 2001; Hall et al. 2004; S Huang, unpubl.). With the exception of nucleolin, none of the ribosome synthesis machinery is detected in the PNC. In an attempt to understand the function of the PNC, we previously used BrdU pulse-labeling for $5 \mathrm{~min}$ and found the PNC to be enriched with newly synthesized RNA. PNC-associated newly synthesized RNA is of Pol III origin and is unaffected by the inhibition of Pol I or II transcription (Huang et al. 1998). These observations led to the obvious question of whether the PNC is the site of synthesis of PNC-enriched RNAs. Efforts from AG Matera et al. (unpubl.) and our laboratory found that PNC is not associated with the genes that transcribe (mitochondrial RNA processing) MRP RNA, RNase P RNA, or hY1 RNA, demonstrating that the PNC is unlikely the site of transcription that synthesizes these RNAs.

If these RNAs are not synthesized in the PNC, with what are they interacting in the PNC, and what might be the function of these interactions? To begin to address these questions, we have examined whether the ribozyme in the PNC is associated with its well-known protein subunits that complete the endonuclease activity of the RNase MRP or P. These subunits were not found to be enriched in the PNC, as are their RNA components, suggesting that MRP RNA and RNase P RNA are not in their characterized RNase complexes when they are associated with the PNC (S Huang, unpubl.). These findings, together with the observation that the PNC is enriched with RNA-binding proteins implicated in Pol II RNA processing, begs the possibility that the PNC-associated RNA and proteins may represent novel protein-RNA complexes.

To determine whether PTB interacts with MRP RNA in vivo, we analyzed the presence of MRP RNA in PTB immunoprecipitates (IPs). The results show that IP PTB coprecipitates MRP RNA specifically (Pollock et al., in press). To evaluate whether PTB directly binds MRP RNA, in vitro gel-shift assays were performed with purified recombinant PTB and in vitro synthesized MRP RNA. Many trials did not reveal direct binding between PTB and MRP RNA (Pollock et al., in press). However, it cannot be excluded that they do interact directly, which may not take place under in vitro experimental conditions.

To further evaluate the interaction, we set up a semi-in vitro assay, based on the principle that positively charged RNA-binding proteins migrate extremely slowly to the anode in native polyacrylamide gels. If there is a specific association between an RNA and a protein (directly or indirectly), addition of the RNA to HeLa nuclear extracts should decrease the net positive charge following certain interactions between the RNA and RNA-binding proteins and thus increase mobility of the RNA-binding protein in native polyacrylamide gels. When HeLa nuclear extracts are resolved on a native acrylamide gel followed by western blot analyses for PTB, La, or CUGBP (PI 9.21, 6.68, and 8.71 , respectively), the majority of these proteins fail to migrate far in the gel (Fig. 3A,B,D; NE alone, lanes 1). In addition to being high in positive charge, these proteins are present in a spliceosome complex of large molecular mass in vivo (Savkur et al. 2001; Charlet et al. 2002; Wag- ner and Garcia-Blanco 2002). Some of the proteins representing the endogenous protein-RNA complexes are observed migrating into the gel.

RNase MRP, P RNA, or SRP RNA generated by in vitro transcription were added to HeLa nuclear extracts and incubated for $20 \mathrm{~min}$ followed by a separation on a $6 \%$ native polyacrylamide gel. When the SRP, MRP, or H1 RNAs were added, a large portion of unbound PTB then migrated into the gel, forming a discrete band that is not observed in the nuclear extract alone (Fig. 3A, lanes 5, 6, and 8). To further evaluate whether the change in PTB mobility is indeed due to specific interactions between the added RNA and PTB, we have also evaluated the effect of RNAs with known PTB-binding sites and those without pyrimidine-rich sequences on PTB mobility. When an RNA fragment ( $\beta$ tropomyosin $[\beta \mathrm{TM}]$ ) known to bind PTB (Grossman et al. 1998) was added into the nuclear extract, a similar increase in mobility of PTB was observed (Fig. 3A, lane 4). However, addition of transfer RNA (tRNA) or granular component (GC)-rich RNA to nuclear extracts did not change PTB mobility in the native gel (Fig. 3A, lanes 2 and 3). Furthermore, treatment of the PTB-RNA complex with RNase A reduced the mobility of the complex, although the reduction is not complete, possibly due to partial protection of the RNA at the regions of protein-RNA interactions (Fig. 3A, lane 7). To further validate the assay, we tested the mobility of the La protein, a known RNA Pol III-binding protein (for review, see Maraia 2001). With a PI of 6.68, a substantial amount of La protein migrates in the native gel (Fig. 3B, lane 1) without addition of RNAs into the nuclear extracts. Following incubation with small RNAs shown to bind La, discrete fast-migrating bands were observed on the native gels (Fig. 3B, lanes 3-5). However, addition of an RNA that does not bind La had no effect on the migration of La (Fig. $3 \mathrm{~B}$, lane 2). Altogether, these findings indicate that the mobility shift of PTB on the native gels by incubation with PNC-associated RNAs is a result of specific interactions between PTB and these RNAs.

The results of these studies further support the hypothesis that PTB interacts with MRP RNA, RNase P RNA, and SRP RNAs that are enriched in the PNC. However, it remains to be clarified whether these interactions are direct or indirect and whether they take place in the PNC.

\section{MATERIAL AND METHODS}

\section{Indirect Immunofluorescence}

Cells were fixed in freshly prepared $4 \%$ paraformaldehyde in PBS for $15 \mathrm{~min}$ and then washed three times for $10 \mathrm{~min}$ each in phosphate-buffered saline (PBS). The cells were then permeabilized with $0.5 \%$ Triton X-100 (in PBS) for $5 \mathrm{~min}$ and washed three times for $10 \mathrm{~min}$ each in PBS. The fixed permeabilized cells were then incubated for $1 \mathrm{~h}$ at room temperature with anti-PTB antibody SH54 (1:200). Cells were rinsed three times for $10 \mathrm{~min}$ each in PBS and then incubated with fluorescein isothiocyanate (FITC)-conjugated goat antimouse antibody (1:200) (Vector Laboratories, Inc., Burlingame, CA) for $1 \mathrm{~h}$ at room temperature followed by three 10-min washes 


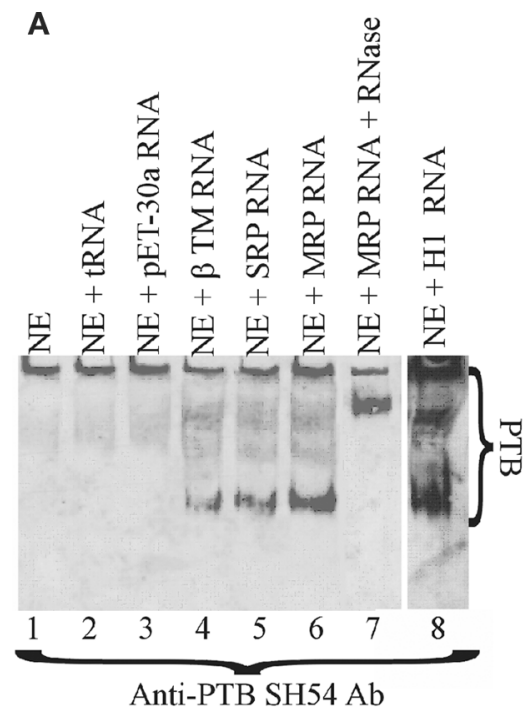

B
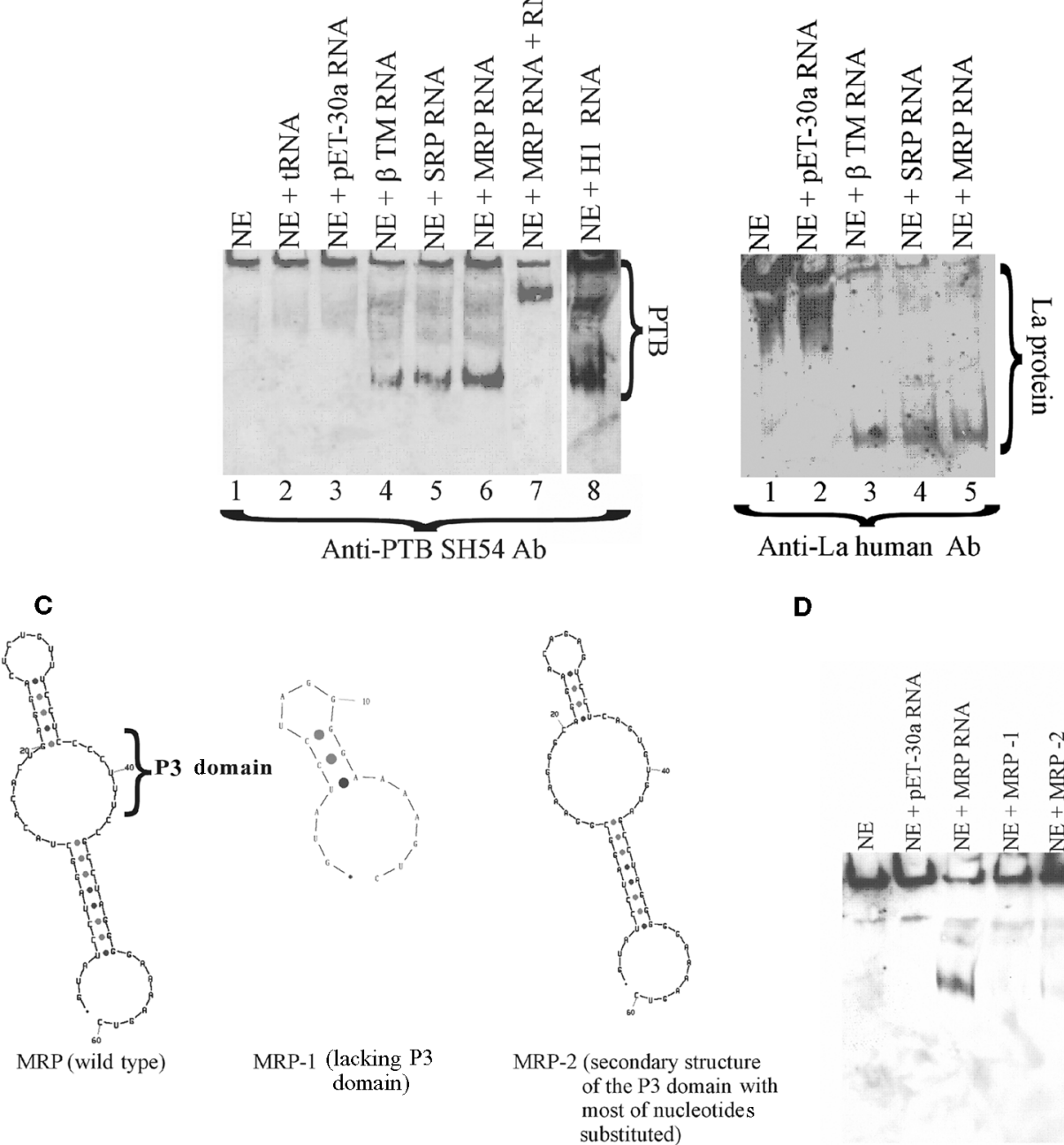

D

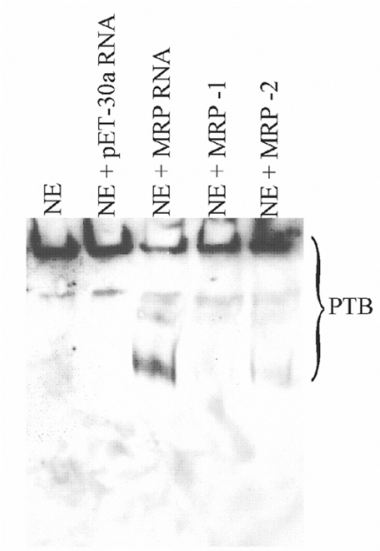

Figure 3. Subset of PNC-associated RNA enhances the mobility of PTB in native acrylamide gels. HeLa nuclear extracts were incubated with in vitro-transcribed RNA, as indicated. The complex was then resolved on $6 \%$ acrylamide gel followed by western blot analysis for PTB $(A)$ or La protein $(B)$. The P3 domain of MRP RNA is required for interactions with PTB. (C) Image of the P3 domain structure of wild-type MRP RNA, mutant MRP-1 RNA lacking the nucleotides 23-62 (P3 domain), or mutant MRP-2 RNA in which nucleotides in the loop regions of the $\mathrm{P} 3$ domain were substituted. $(D)$ Gel-shift assays were preformed using HeLa nuclear extracts incubated with wild-type MRP RNA, mutant MRP-1 RNA, or mutant MRP-2 RNA followed by western blot analyses for PTB protein.

in PBS. The coverslips were mounted onto glass slides with mounting medium containing $90 \%$ glycerol in PBS adjusted to $\mathrm{pH} 8.0$ with 0.2 - $\mathrm{M}$ bicarbonate buffer and 1 $\mathrm{mg} / \mathrm{mL}$ paraphenylenediamine (Sigma-Aldrich, St. Louis, MO) as an antifade agent. Cells were observed on a Zeiss Axiovert 135 microscope, and images were acquired with a SenSys cooled charge-coupled device (CCD) camera (Photometrics, Tucson, AZ) using MetaMorph Version 4.5 software (Universal Imaging Corporation Ltd., West Chester, PA).

\section{ESI}

Hela cells were similarly grown and prepared as indicated in the indirect immunofluorescence section above.
Instead of the FITC secondary antibody, however, cells were incubated with a gold-conjugated donkey antimouse antibody (1:100) (Electron Microscopy Sciences, No. 25811) for $1 \mathrm{~h}$ followed by three 5-min PBS washes. Cells were postfixed in $2 \%$ glutaraldehyde for $5 \mathrm{~min}$, followed by three 5-min PBS washes and one 5-min wash in double distilled water (Invitrogen Canada Inc., No. 15230-162). The labeled gold particles were silver enhanced for $15 \mathrm{~min}$ at $37^{\circ} \mathrm{C}$ (Electron Microscopy Sciences, No. 25521), followed by four 5 -min washes in double distilled water and dehydration through a graded series of ethanol dilutions: $30 \%, 50 \%, 70 \%, 90 \%$, and $100 \%$ for 30 min each, followed by two more changes of $100 \%$ ethanol for $10 \mathrm{~min}$ each. Dehydrated cells were embedded in Quetol 651 as described in Ahmed et al. 2009. The 70-nm sections of 
cells were obtained with an Ultracut UCT ultramicrotome (Leica Microsystems Inc.) and picked up on electron microscope (EM) grids. The sections on grids were then carbon coated to prevent deformation in the electron beam. Electron micrographs were obtained at $200 \mathrm{kV}$ on a TEM (FEI Technai 20) equipped with an ESI (Gatan). A lowmagnification image containing the entire nucleus in the imaging field was first obtained to locate the dense region of gold particles at the nucleolar periphery corresponding to the PNC. At higher magnifications, this region was then imaged as follows: Net phosphorus images were obtained by the ratio of images recorded at 120 electron volts (preedge) and 150 electron volts (postedge), corresponding to before and after the phosphorus ionization edge $\left(\mathrm{L}_{\mathrm{II}}, \mathrm{III}, 132\right.$ electron volts), respectively (Bazett-Jones and Hendzel 1999). Net nitrogen images were obtained by the ratio of pre-edge and postedge images recorded at 385 and 415 electron volts, respectively. The resultant elemental maps were then processed to discern chromatin-based structures from nonchromatin-based protein-rich structures: To eliminate the contribution of the chromatin-based signal in the nitrogen map, a phosphorus signal is subtracted, and the resultant difference map is thresholded to zero in chromatin regions. The phosphorus signal corresponding to chromatin is indicated with yellow, and the nitrogen signal corresponding to protein is indicated with cyan.

\section{RNA-Binding Protein Gel-Shift Assays}

Nuclear extracts were prepared from HeLa cells (Paillard et al. 2003) and SRP, $\beta$ TM, and pET-30a (GC-rich) RNA transcripts were generated by in vitro transcription assays using T7 RNA polymerase, whereas wild-type MRP RNA and mutant MRP-1 and MRP-2 RNA transcripts were generated with T3 RNA polymerase (Roche Applied Sciences). Nuclear extracts (5 $\mu \mathrm{g})$ were incubated with $1 \mathrm{fmol}$ of RNA transcript (MRP, SRP, $\beta$ TM, or pET30a) for $20 \mathrm{~min}$ (Anand et al. 2001). For RNase treatment, complexes were incubated with RNase A $(1 \mu \mathrm{g} / \mathrm{mL})$ for $30 \mathrm{~min}$ at $37^{\circ} \mathrm{C}$. Reactions were then resolved on a $6 \%$ native acrylamide gel and transferred to nitrocellulose membrane. Western blot analyses were performed with anti-PTB antibody (SH54).

\section{Tissue Samples}

Paraffin-embedded colorectal and breast tissue samples were obtained from Northwestern University Medical School and the Cooperative Human Tissue Network at the University of Pennsylvania Medical Center. We used 176 colorectal samples for the analysis. Cancer tissue samples were grouped using the American Joint Committee on Cancer TNM staging scheme. Some patients with primary tumors had corresponding clinical and follow-up data including recurrence and distal metastases.

\section{Immunohistochemistry on Tissue Sections}

To examine various colorectal and breast tissue samples for PNC prevalence, a standard detection protocol was used. The PNC can be detected by immunocytochemical labeling using an antigen retrieval protocol with monoclonal antibodies (SH54) that we have developed to specifically recognize the key PNC-associated protein PTB. PTB is an RNA-binding protein that is highly concentrated in PNC and serves as a marker for the PNC. The basic protocol involves deparaffinization of 4-5- $\mu \mathrm{m}$ paraffin-embedded tissue sections in xylene and graded alcohols followed by microwave antigen retrieval for $2 \mathrm{~min}$ in 10-mM citric acid buffer ( $\mathrm{pH}$ 6.0) before applying the conventional immunolabeling protocol (including incubation with the primary antibody and subsequent incubation with an avidin-conjugated secondary antibody). Signals are detected using horseradish peroxidase-conjugated biotin that binds to avidin (Vector Laboratories, Inc., Burlingame, CA). The enzyme converts chromogen 3,3'-diaminobenzidine (Sigma-Aldrich, St. Louis, MO) into dark precipitates in the presence of hydrogen peroxide. Sections are not counterstained. Signals are visualized using light microscopy (Nikon Eclipse E800), and images are captured through a $60 \times$ objective.

\section{Statistical Analysis}

Differences in PNC prevalence among clinical and pathologic variables were assessed by analysis of variance. These variables were based on tissue microarray (TMA) staging, histopathological grading, and lymph node involvement. Survival analyses evaluated the prognostic value of the PNC. In a univariate survival analysis, the prognostic value of the PNC was conducted with three types of patient outcomes. The outcomes selected for the study included disease-specific survival (defined as time to death from disease), disease-free survival (defined as time to local recurrence or distant metastasis), and overall survival (defined as time to death from any cause). For the univariate survival analysis, the mean survival times and their 95\% confidence intervals were calculated via KaplanMeier estimation. Differences in Kaplan-Meier survival curves among the clinical and pathologic variables were evaluated with the log-rank test for 1 degree of freedom.

\section{CONCLUSION}

The PNC is a distinct nonmembrane-bound subnuclear structure that only forms at advanced stages of cellular transformation. PNC prevalence closely associates with the metastatic capability of a given cell population, and a high PNC prevalence in primary tumors is linked to poor patient outcome. PNC formation or disassembly does not reflect cellular stresses or changes in cellular metabolic or differentiation conditions. The PNC is nucleated on a DNA locus or loci that has active transcriptional activities in PNC-containing cells. A subset of Pol III, noncoding RNA, and a group of RNA-binding proteins that have been primarily implicated in Pol II RNA processing have been shown to be enriched in the PNC. Based on our studies, we propose a working model (Fig. 4) in which novel RNA-protein complexes nucleate at the PNC-containing DNA locus or loci. These complexes may have an important role in reg- 


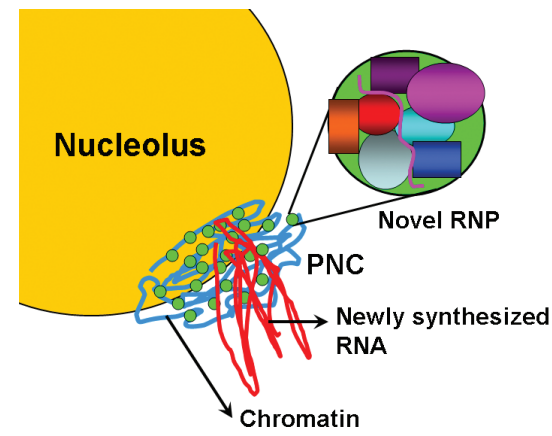

Figure 4. Diagram of the working model in which the novel RNP has a role in regulating gene expression of the DNA loci that nucleate the PNC.

ulating the gene expression of these loci either transcriptionally or posttranscriptionally. The formation of the PNC may reflect the changes in this regulation in cancer cells. Studies are underway to test this hypothesis.

\section{ACKNOWLEDGMENTS}

We are grateful for the support from the National Institutes of Health, General Medicine for S.H. (R01 GM078555-01A1). Research was funded by operating grants from the Canadian Institutes of Health Research and the Natural Sciences and Engineering Research Council to D.P.B.-J., who holds the Canada Research Chair in Molecular and Cellular Imaging.

\section{REFERENCES}

Ahmed K, Li R, Bazett-Jones DP. 2009. Electron spectroscopic imaging of the nuclear landscape. Methods Mol Biol 464: 415423.

Anand A, Villella A, Ryner LC, Carlo T, Goodwin SF, Song HJ, Gailey DA, Morales A, Hall JC, Baker BS, et al. 2001. Molecular genetic dissection of the sex-specific and vital functions of the Drosophila melanogaster sex determination gene fruitless. Genetics 158: 1569-1595.

Bazett-Jones DP, Hendzel MJ. 1999. Electron spectroscopic imaging of chromatin. Methods 17: 188-200.

Charlet BN, Savkur RS, Singh G, Philips AV, Grice EA, Cooper TA. 2002. Loss of the muscle-specific chloride channel in type 1 myotonic dystrophy due to misregulated alternative splicing. Mol Cell 10: 45-53.

Dellaire G, Nisman R, Bazett-Jones DP. 2004. Correlative light and electron spectroscopic imaging of chromatin in situ. Methods Enzymol 375: 456-478.

Gall JG. 2003. The centennial of the Cajal body. Nat Rev Mol Cell Biol 4: 975-980.

Grossman JS, Meyer MI, Wang YC, Mulligan GJ, Kobayashi R, Helfman DM. 1998. The use of antibodies to the polypyrimidine tract binding protein (PTB) to analyze the protein components that assemble on alternatively spliced pre-mRNAs that use distant branch points. RNA 4: 613-625.

Hall MP, Huang S, Black DL. 2004. Differentiation-induced colocalization of the KH-type splicing regulatory protein with polypyrimidine tract binding protein and the c-src pre-mRNA. Mol Biol Cell 15: 774-786.

Huang S, Deerinck TJ, Ellisman MH, Spector DL. 1998. The perinucleolar compartment and transcription. J Cell Biol 143: 35-47.
Huttelmaier S, Illenberger S, Grosheva I, Rudiger M, Singer RH, Jockusch BM. 2001. Raver1, a dual compartment protein, is a ligand for PTB/hnRNPI and microfilament attachment proteins. J Cell Biol 155: 775-786.

Kamath RV, Thor AD, Wang C, Edgerton SM, Slusarczyk A, Leary DJ, Wang J, Wiley EL, Jovanovic B, Wu Q, et al. 2005. Perinucleolar compartment prevalence has an independent prognostic value for breast cancer. Cancer Res 65: 246-253.

Lamond AI, Earnshaw WC. 1998. Structure and function in the nucleus. Science 280: 547-553.

Lamond AI, Spector DL. 2003. Nuclear speckles: A model for nuclear organelles. Nat Rev Mol Cell Biol 4: 605-612.

Lee B, Matera AG, Ward DC, Craft J. 1996. Association of RNase mitochondrial RNA processing enzyme with ribonuclease $\mathrm{P}$ in higher ordered structures in the nucleolus: A possible coordinate role in ribosome biogenesis. Proc Natl Acad Sci 93: 11471-11476.

Liu JL, Wu Z, Nizami Z, Deryusheva S, Rajendra TK, Beumer KJ, Gao H, Matera AG, Carroll D, Gall JG. 2009. Coilin is essential for Cajal body organization in Drosophila melanogaster. Mol Biol Cell 20: 1661-1670.

Maraia RJ. 2001. La protein and the trafficking of nascent RNA polymerase III transcripts. J Cell Biol 153: F13-F18.

Matera AG, Frey MR, Margelot K, Wolin SL 1995. A perinucleolar compartment contains several RNA polymerase III transcripts as well as the polypyrimidine tract-binding protein, hnRNP I. $J$ Cell Biol 129: 1181-1193.

Norton JT, Pollock CB, Wang C, Schink JC, Kim JJ, Huang S. 2008. Perinucleolar compartment prevalence is a phenotypic pancancer marker of malignancy. Cancer 113: 861-869.

Norton JT, Wang C, Gjidoda A, Henry RW, Huang S. 2009. The perinucleolar compartment is directly associated with DNA. $J$ Biol Chem 284: 4090-4101.

Oakes M, Siddiqi I, Vu L, Aris J, Nomura M. 1999. Transcription factor UAF, expansion and contraction of ribosomal DNA (rDNA) repeats, and RNA polymerase switch in transcription of yeast rDNA. Mol Cell Biol 19: 8559-8569.

Paillard L, Legagneux V, Beverley Osborne H. 2003. A functional deadenylation assay identifies human CUG-BP as a deadenylation factor. Biol Cell 95: 107-113.

Pederson T. 1998. The plurifunctional nucleolus. Nucleic Acids Res 26: 3871-3876.

Pollock C, Daily K, Nguyen VT, Wang C, Lewnadowska M, Bensaude O, Huang S. 2010. Characterization of MRP RNA-protein interactions within the perinucleolar compartment. Mol Biol Cell (in press).

Savkur RS, Philips AV, Cooper TA. 2001. Aberrant regulation of insulin receptor alternative splicing is associated with insulin resistance in myotonic dystrophy. Nat Genet 29: 40-47.

Spector DL. 2003. The dynamics of chromosome organization and gene regulation. Annu Rev Biochem 72: 573-608.

Strzelecka M, Trowitzsch S, Weber G, Luhrmann R, Oates AC, Neugebauer KM. 2010. Coilin-dependent snRNP assembly is essential for zebrafish embryogenesis. Nat Struct Mol Biol 17: 403-409.

Timchenko LT, Miller JW, Timchenko NA, DeVore DR, Datar KV, Lin L, Roberts R, Caskey CT, Swanson MS. 1996. Identification of a (CUG)n triplet repeat RNA-binding protein and its expression in myotonic dystrophy. Nucleic Acids Res 24: 4407-4414.

Tucker KE, Berciano MT, Jacobs EY, LePage DF, Shpargel KB, Rossire JJ, Chan EK, Lafarga M, Conlon RA, Matera AG. 2001. Residual Cajal bodies in coilin knockout mice fail to recruit Sm snRNPs and SMN, the spinal muscular atrophy gene product. $J$ Cell Biol 154: 293-307.

Wagner EJ, Garcia-Blanco MA. 2002. RNAi-mediated PTB depletion leads to enhanced exon definition. Mol Cell 10: $943-$ 949.

Wang C, Politz JC, Pederson T, Huang S. 2003. RNA polymerase III transcripts and the PTB protein are essential for the integrity of the perinucleolar compartment. Mol Biol Cell 14: 2425-2435 


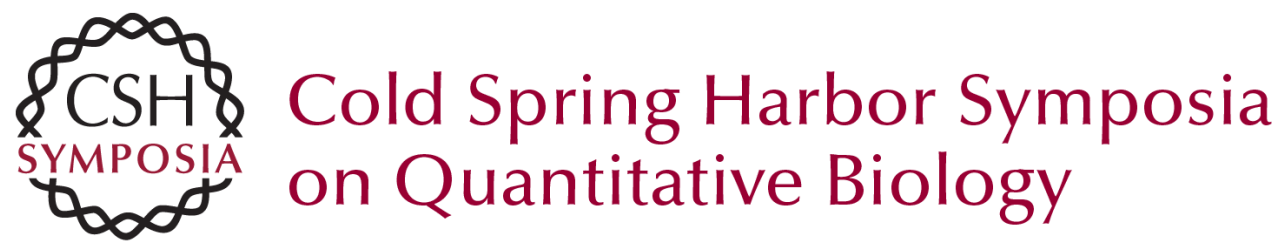

\section{Structure and Function of the Perinucleolar Compartment in Cancer Cells}

A. Slusarczyk, R. Kamath, C. Wang, et al.

Cold Spring Harb Symp Quant Biol 2010 75: 599-605 originally published online February 2, 2011

Access the most recent version at doi:10.1101/sqb.2010.75.026

References This article cites 29 articles, 14 of which can be accessed free at:

http://symposium.cshlp.org/content/75/599.full.html\#ref-list-1

\section{License}

Email Alerting Receive free email alerts when new articles cite this article - sign up in the box at the Service top right corner of the article or click here. 